\section{Orthopädie und Europa}

Die EU wächst schnell, in manchen Bereichen unter politischem Erfolgsdruck zu schnell und dysharmonisch.

So hat die Europäische Kommission (EK) mit einigen zentral- und osteuropäischen Ländern (Bulgarien, Ungarn, Polen, Rumänien, Slowakei und Tschechien) im Vorgriff auf ihren angestrebten EU-Beitritt eine Assoziations-Vereinbarung unterzeichnet, die den Ärzten dieser Länder (Arbeitern und Dienstleistern allg.) ab II./98 Freizügigkeit und Niederlassungsfreiheit in der EU gewährt. Dies verpflichtet die Aufnahmeländer, Diplome, Prüfungzeugnisse und Befähigungsnachweise dieser Kollegen als gleichrangig mit den landeseigenen anzuerkennen, obwohl diese Äquivalenz bei unbekannten WB-Bedingungen nicht erwiesen ist. Für die Stammländer der EU wurden Freizügigkeit und Niederlassungsrecht schon 1975 mit der Richtlinie 75/362 EWG geregelt. Die politische Vorgabe war dabei ein gleich hoher Standard der ärztlichen Versorgung der Bevölkerung in allen Mitgliedsländern. Die Gewährleistung dieser Vorgabe sollte mit den in der Richtlinie festgelegten Mindest-WB-Zeiten erreicht werden.

$\mathrm{Da}$ allein über diese Festlegung ein einheitlicher und hoher Qualitätsstandard nicht gewährleistet werden kann, ist offensichtlich. Leider beschränkt sich aber auch die novellierte Richtlinie 93/16 EWG immer noch auf die Festlegung von Mindest-WB-Zeiten, die teilweise sogar unter den nationalen Anforderungen liegen.

Die Europäische Vereinigung der Fachärzte (UEMS), ihre Gebietssektionen und die von diesen gegründeten European Boards bemühen sich seit Jahren darum, daß die Mindest-WB-Zeiten in den Richtlinien den tatsächlichen Gegebenheiten angepaßt und darüberhinaus auch der Inhalt der WB und ihre Strukturierung in die Richtlinien aufgenommen werden. Diese Gremien arbeiten auch intensiv daran, die WB-Ordnung der EU-Länder zu harmonisieren, um einen einheitlichen Qualitätsstandard ärztlicher Tätigkeit in der EU zu erreichen.

Die Einflußmöglichkeiten der UEMS auf die Beschlüsse der EK sind jedoch begrenzt auf die Erstellung von Empfehlungen und Leitlinien, die über den Ständigen Ausschu $\beta$ der Arzteschaft der EU an den Beratenden Ausschu $\beta$ der EU für die Ärztliche Aus- und Weiterbildung gelangen. Erst mit diesem Ausschuß ist in der europäischen Hierarchie die EU-Behörde erreicht. Er wurde vom Ministerrat mit der Aufgabe eingesetzt, ,, an der Sicherstellung der ärztlichen Aus- und Weiterbildung auf einem vergleichbar hohen Standard innerhalb der Gemeinschaft mitzuwirken“. Die EK ist verpflichtet, sich mit den Empfehlungen des Beratenden Ausschusses auseinanderzusetzen, sie ist jedoch nicht an diese Empfehlungen gebunden. Die Kommission entscheidet völlig unabhängig darüber, ob sie die Empfehlungen des Beratenden Ausschusses an das Europäische Parlament, an den Ministerrat und an die zuständigen Behörden der Mitgliedstaaten weiterleitet. Da berufliche Belange also nur auf diesem Weg an die EK gelangen, sind sie gegenüber politischen Gesichtspunkten in jeder Hinsicht nachrangig. Es kommt hinzu, daß die EK derzeit keine rechtlichen Möglichkeiten hat, in bestehendes nationales Gesundheitsreicht einzugreifen.

\footnotetext{
Z. Orthop. 135 (1997) 477-478

(C) 1997 F. Enke Verlag Stuttgart
}

Der Gesundheitsschutz ist in den europäischen Verträgen nicht speziell verankert. Die Gesundheitssysteme der Mitgliedsstaaten sind aus politischen Gründen für die EK unantastbar. Im Gesundheitswesen beschränken sich die Initiativen der EK erklärtermaßen auf die Förderung einer kohärenten Politik im Bereich der Volksgesundheit.

Die aus ärztlicher und europäischer Sicht erforderliche Harmonisierung der ärztlichen Aus- und Weiterbildung bleibt also vorrangig Aufgabe der UEMS, ihrer Sektionen und der European Boards mittels Empfehlungen und Leitlinien. Ihre Umsetzung in Landesrecht ist derzeit aber nur über die nationalen Gesundheitsbehörden möglich.

Die in der Richtlinie 93/16 EWG für die Orthopädie geforderte Mindest-WB-Zeit von 5 Jahren wird inzwischen von allen 17 Mitgliedsländern der UEMS erfüllt, von sieben Ländern überschritten, von dreien sogar erheblich (GB u. SF $3 \mathrm{~J}$., N $4 \frac{1}{2} \mathrm{~J}$.). In SF und $\mathrm{N}$ ist die orthopädische WB erst nach voller Ableistung der chirurgischen WB (6 J.) möglich. In den übrigen Ländern wird eine unterschiedlich lange chirurgische WB-Zeit gefordert: 4 Jahre in GB, 3 Jahre in B, CH u. IRL, 2 Jahre in A, DK, $\mathrm{E}, \mathrm{L}, \mathrm{NL}, 1^{1} / 2$ Jahre in F, 1 Jahr in D, GR u. P.

Im Bereich der WB-Inhalte, der Strukturierung der WB und der Kontrolle ihrer ordnungsgemäßen Erfüllung durch Einsatz eines Tutors, Führung eines Log-Buches, Zwischen- und Abschlußexamina sind die Unterschiede nicht weniger groß.

In 9 Ländern werden Zwischenexamina (teilweise jährlich) und ein Abschlußexamen mit unterschiedlicher Gestaltung durchgeführt (teils nur mündlich, teils mündlich, schriftlich und praktisch). Ohne Examen wird das FA-Diplom in DK, E und S erteilt; die WB-Struktur ist aber in diesen Ländern durch Curriculum geregelt. In 10 Ländern sind vom Kandidaten Tätigkeitsnachweise in einem LogBuch zu führen, dessen Eintragungen vom Tutor oder dem ermächtigten Lehrer zu bestätigen sind, und das der diplomierenden Institution vorzulegen ist. Ein Tutor (auch Koordinator) wird dem Kandidaten in 8 Ländern zur Seite gestellt. In 5 Ländern sind vorgeschriebene Spezialkurse zu absolvieren.

In der BRD ist die Nachweispflicht beschränkt auf die Vorlage des bestätigten Operationskataloges und des FAZeugnisses. Das abschließende FA-Examen ist inhaltlich nicht strukturiert, als formale Vorgabe gilt nur sein Charakter als Kollegialgespräch mit einer Dauer von längstens 30 Minuten. Als Evaluation mit bundeseinheitlichem Bewertungsmaßstab und Vergleichbarkeit der Ergebnisse kann das deutsche FA-Examen daher nicht gelten.

Durch die arbeitsrechtliche Situation in der BRD (jede Ass.-Stelle ist eine WB-Stelle) ist die Zahl der vom ermächtigten Arzt weiterzubildenden Assistenten in vielen Fällen so groß, daß die individuelle Führung und Betreuung der Kandidaten nicht mehr im erforderlichen Umfang möglich ist. In Ländern mit quotierten WB-Stellen ist das Kandidaten-Lehrer-Verhältnis zwangsläufig günstiger, teilweise auch vorgeschrieben.

Die orthopädische WB in der BRD ist daher im europäischen Vergleich nach Anspruch und Ergebnis unterschiedlich zu beurteilen. Die in der deutschen WBO und ihren Richtlinien gestellten Qualitäts-Anforderungen sind zweifellos hoch angesiedelt und deshalb für andere europäische Länder beispielhaft. Bei der Evaluation des WB-Ergebnisses fällt die BRD aber deutlich ab. 
Wegen der schleppenden Fortschritte in der Harmonisierung der WB-Bedingungen in Europa wurden auf Empfehlungen der UEMS von den Gebiets-Sektionen European Boards gegründet. Sie haben die Aufgabe, an der Gestaltung und Harmonisierung der WB unter Betonung fachlich-wissenschaftlicher Gesichtspunkte mitzuwirken. Einige Boards haben in forscher Vorwärtsstrategie Struktur und Bedingungen für ein europäisches Examen ausgearbeitet, organisiert und auch schon durchgeführt (Anästhesie, Urologie, Ophthalmologie, Physikalische Medizin). Dieses Examen ist kein Ersatz für das nationale FA-Examen; es kann sogar nur von Kandidaten abgelegt werden, die bereits einen nationalen FA-Titel erworben und schon mehrere Jahre in ihrem Fachgebiet praktiziert haben. Trotz Freiwilligkeit der Teilnahme wurden diese Examen europaweit gut angenommen. Die erfolgreichen Kandidaten erhalten mit dem Diplom keinen Rechtstitel, wohl aber einen fachlichen Qualifikationsnachweis, der für die berufliche Karriere in mancher Hinsicht nützlich sein kann und auch sein wird.

Der European Board of Orthopaedics (EBO) hat kein Examen in seine Satzung aufgenommen, dann wohl aber die Möglichkeit verankert, ein Qualitätszertifikat zu vergeben (Recognition of Quality in Orthopaedics and Traumatology) durch eine noch nicht näher definierte oder organisierte Evaluation.

Die von der WB-Kliniken zu erbringenden Leistungen als Voraussetzung und Gewähr für eine WB-Qualität auf hohem Niveau (Arbeitsbedingungen, Regelarbeitszeit, Möglichkeiten und Bedingungen für theoretische und wissenschaftliche Betätigung innerhalb und außerhalb der Regeldienstzeiten, Supervision der Kandidaten u.a.) werden sehr unterschiedlich bewertet und überprüft. In 9 Ländern der EU werden Klinik-Visitationen in regelmäßigen Abständen durch eine beauftragte Kommission nach einem geregelten Programm durchgeführt; die weitere Lizensierung der Klinik ist abhängig vom Ergebnis dieser Visitation. In 2 Ländern erfolgt die Visitation auf freiwilliger Basis und auf Antrag der Klinik, wobei die Kliniken offensichtlich Interesse an der damit verbundenen Qualitäts-Zertifizierung haben.

Bei diesem Krankenhaus-Check wird auch das Wirken der Ärzte in ihrer Funktion als Lehrer evaluiert; diese Bewertung berücksichtigt unter strenger Wahrung der Diskretion auch die Beurteilung der Kandidaten selber. Die Kosten der Visitationen werden teilweise von der National Medical Association getragen (S), teilweise ausschließlich von den Klinken selber. Die Organisation und die Bestellung der Kommission aus dem Kreise anerkannter Experten erfolgt durch die Wissenschaftlichen Gesellschaften, die auch das Prüfergebnis der Kommission entgegennehmen und die entsprechenden Konsequenzen daraus ziehen und vollziehen.

Die berechtigten Interessen werden auf europäischer Ebene von der Permanent Working Group of Junior Hospital Doctors (PWG) vertreten; sie ist auch in der Orthopädischen Sektion mit einem Delegierten im Beobachterstatus präsent.

Die ärztliche Fortbildung wird in allen Mitgliedsländern der EG gleichermaßen als Voraussetzung und Garantie für eine ärztliche Patienten-Versorgung auf hohem Niveau bewertet. Ihre Strukturierung auf freiwilliger oder verpflichtender Basis ist jedoch unterschiedlich oder auch nicht geregelt. Es befassen sich auch unterschiedliche Behörden, Institutionen und freie Organisationen mit dieser Thematik. In einigen Ländern der EU gibt es aber schon strikte Regeln, teilweise von Behörden vorgeschrieben und überwacht, teilweise aber auch ausschließlich von Berufsorganisationen organisiert und praktiziert.

Überhaupt sind die wissenschaftlichen Gesellschaften und Berufsverbände in vielen EU-Ländern die Promotoren für die Realisierung sinnvoller Maßnahmen zur Qualitätssicherung ärztlicher Arbeit. Nicht selten handeln sie unter dem Druck, behördlichen Verfügungen zuvorzukommen und in der erklärten Absicht, die Kontrollfunktionen in Händen der Berufsorganisationen zu halten.

Der European Board of Radiology hat zusammen mit der European Association of Radiology eine Satzung beschlossen, in der Umfang, Organisation, Durchführung und Kontrolle der ärztlichen Fortbildung in der Radiologie geregelt sind. Im nationalen und europäischen Bereich kann sie zwar nur als Empfehlung und Leitlinie gelten, unter den gegebenen Umständen ist sie aber dennoch von Bedeutung.

In der BRD ist die ärztliche Fortbildung als ethische und moralische Verpflichtung in der Berufsordnung $(\mathrm{BO})$ verankert, woraus selbstverständlich auch eine rechtliche Verpflichtung resultiert. Strukturierten Vorschriften für Durchführung und Kontrolle der Fortbildung hat sich der Deutsche Ärztetag bisher aber verweigert. Die BÄK hat zwar bereits 1993 Empfehlungen und Leitlinien zur Organisation und Durchführung der Fortbildung veröffentlicht, ihre Umsetzung in den Landes-Äztekammern ist bisher jedoch nicht erfolgt.

Die von der UEMS und ihren Sektionen erarbeiteten Empfehlungen und Leitlinien zur inhaltlichen und strukturellen Harmonisierung fachärztlicher Tätigkeit in der EG auf einheitlich hohem Qualitätsniveau wurden themenbezogen als Charta publiziert und den nationalen und europäischen Institutionen zugestellt, u.a. sind erschienen: European Training Charter, Charter on CME of medical specialists, Charter on Quality Assurance und Charter on Visitation of Training Centres mit sehr detaillierten Vorschlägen für die praktische Durchführung.

In der BRD fußt die Qualitätssicherung ärztlicher Tätigkeit vorrangig auf den hohen Ansprüchen der BO, wobei die freiheitliche Gestaltung und Ausübung des Berufes vorgeschriebenen Kontrollen vorgezogen wird. Dies ist aber nur solange möglich, wie die erforderliche Freiheit erhalten bleibt. Ihre Beschränkung durch politische Eingriffe ist jedoch zu befürchten. Auch ist zu bezweifeln, daß die Einflußnahme der EU auf die nationalen Gesundheitssysteme dauerhaft auf die Festsetzung der Mindest-WB-Zeit beschränkt bleiben wird. Tendenzen sind erkennbar auch von Seiten der Ärzteschaft - bei einer Novellierung des Vertrages von Maastricht die Kompetenzen der EU im Gesundheitswesen zu erweitern.

Die Allianz Deutscher Orthopäden wäre daher gut beraten, die europäischen Entwicklungstendenzen in die Gestaltung ihrer Zukunftsstrukturen einzubeziehen.

Giselbert Fries, Saarbrücken Delegierter der DGOT in der Orthopädischen Sektion der UEMS und im European Board of Orthopaedics 